\title{
Synchronized Offset Stacking: A Concept for Growing Large-Domain and Highly Crystalline 2D Covalent Organic Frameworks
}

\author{
Florian Auras, ${ }^{\dagger, \ddagger \odot}$ Laura Ascherl, ${ }^{\dagger}$ Amir H. Hakimioun, ${ }^{\S}$ Johannes T. Margraf, ${ }^{\S}$ Fabian C. Hanusch, ${ }^{\dagger}$
} Stephan Reuter, ${ }^{\dagger}$ Derya Bessinger, ${ }^{\dagger}$ Markus Döblinger, ${ }^{\dagger}$ Christina Hettstedt, ${ }^{\dagger}$ Konstantin Karaghiosoff, ${ }^{\dagger}$ Simon Herbert, ${ }^{\dagger}$ Paul Knochel, ${ }^{\dagger} \odot$ Timothy Clark, ${ }^{\S \subseteq}$ and Thomas Bein ${ }^{*}$,

${ }^{\dagger}$ Department of Chemistry and Center for NanoScience (CeNS), University of Munich (LMU), Butenandtstraße 5-13, 81377 Munich, Germany

${ }^{\ddagger}$ Cavendish Laboratory, University of Cambridge, Cambridge CB3 0HE, United Kingdom

${ }^{\S}$ Friedrich-Alexander-University Erlangen-Nürnberg (FAU), Computer-Chemie-Centrum, Nägelsbachstraße 25, 91052 Erlangen, Germany

\section{Supporting Information}

ABSTRACT: Covalent organic frameworks (COFs), formed by reversible condensation of rigid organic building blocks, are crystalline and porous materials of great potential for catalysis and organic electronics. Particularly with a view of organic electronics, achieving a maximum degree of crystallinity and large domain sizes while allowing for a tightly $\pi$-stacked topology would be highly desirable. We present a design concept that uses the 3D geometry of the building blocks to generate a lattice of uniquely defined docking sites for the attachment of consecutive layers, thus allowing us to achieve a greatly improved degree of order within a given average number of attachment and detachment cycles during COF growth. Synchronization of the molecular geometry across

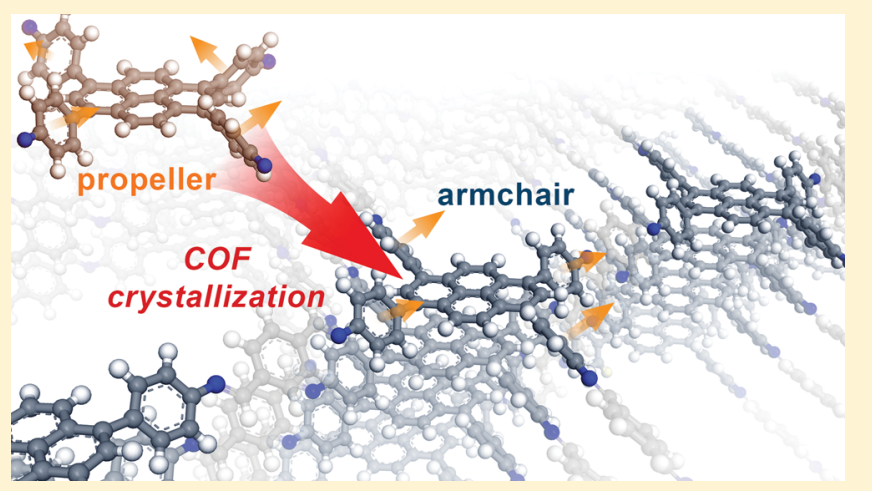
several hundred nanometers promotes the growth of highly crystalline frameworks with unprecedented domain sizes. Spectroscopic data indicate considerable delocalization of excitations along the $\pi$-stacked columns and the feasibility of donoracceptor excitations across the imine bonds. The frameworks developed in this study can serve as a blueprint for the design of a broad range of tailor-made $2 \mathrm{D}$ COFs with extended $\pi$-conjugated building blocks for applications in photocatalysis and optoelectronics.

\section{INTRODUCTION}

Growing extended and high quality crystals of molecular framework materials has remained a challenge, particularly in the case of covalent organic frameworks (COFs), where the connections between the individual building blocks are formed by covalent bonds. COFs are porous long-range ordered materials that have recently attracted considerable scientific attention as candidates for gas storage and separation, ${ }^{1,2}$ catalysis, $^{3,4}$ and as new materials for organic electronics and optoelectronics. $^{5-7}$ In view of the last potential application, COFs that are covalently linked in two dimensions while held together by $\pi$-stacking in the third dimension (referred to as 2D-COFs), are of particular interest. ${ }^{8,9}$ The self-assembled $\pi$-stacked columns that are formed in these materials enable electronic transport across the layers. ${ }^{10,11}$ Additionally, if the linkage between the individual building blocks that constitute the $2 \mathrm{D}$ layers is $\pi$ conjugated, as for example in the case of imine-linked frameworks, the conductivity might be extended to the other two dimensions. The aligned open channels that surround the molecular stacks can be used to alter the properties or extend the functionality of the host material through the incorporation of guest molecules, such as fullerenes, ${ }^{5,12,13}$ tetracyanoquinodimethane, ${ }^{14}$ or iodine. ${ }^{15,16}$

In view of these potential applications it is essential to construct highly crystalline (i.e., a maximum degree of coherent long-range order within a crystal domain) frameworks with large crystal domains and to be able to select from a broad range of specialized building blocks. Possible applications in gas separation or catalysis require fully accessible pores that are not blocked due to stacking faults or amorphous regions. The factor of crystallinity becomes even more important if applications in electronics or optoelectronics are intended, as stacking faults could disrupt the conductive $\pi$-stacked columns, and defect sites and grain boundaries could act as traps and recombination sites for excitons or charges.

Received: September 18, 2016

Published: December 19, 2016 

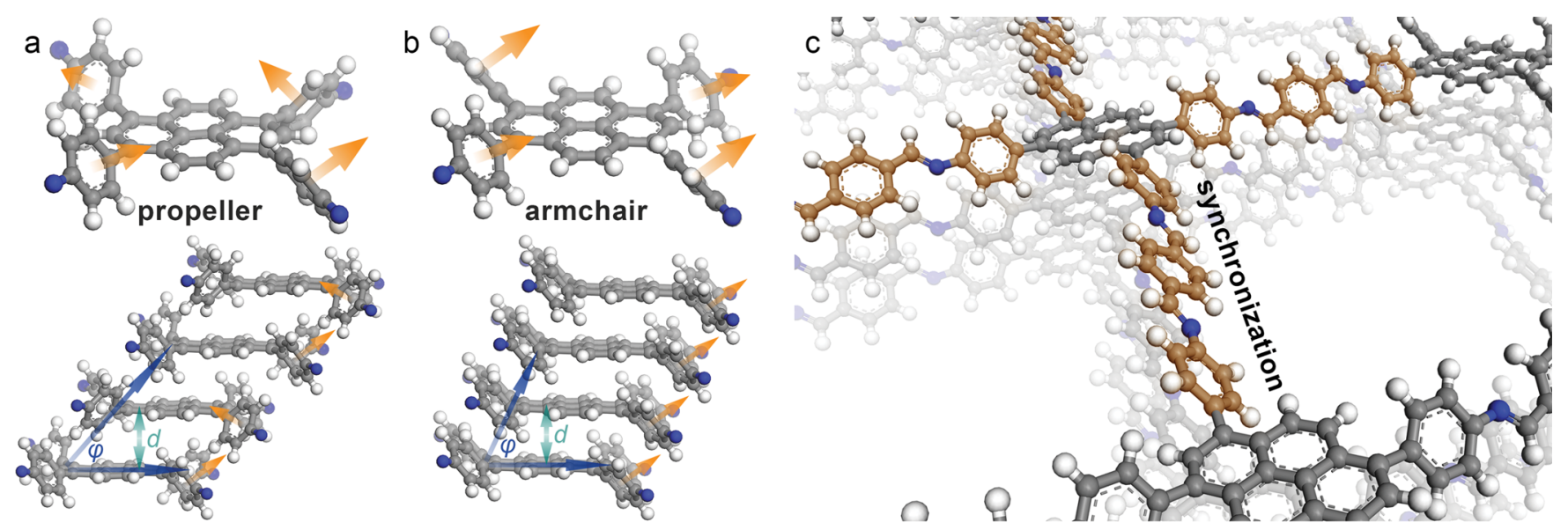

d
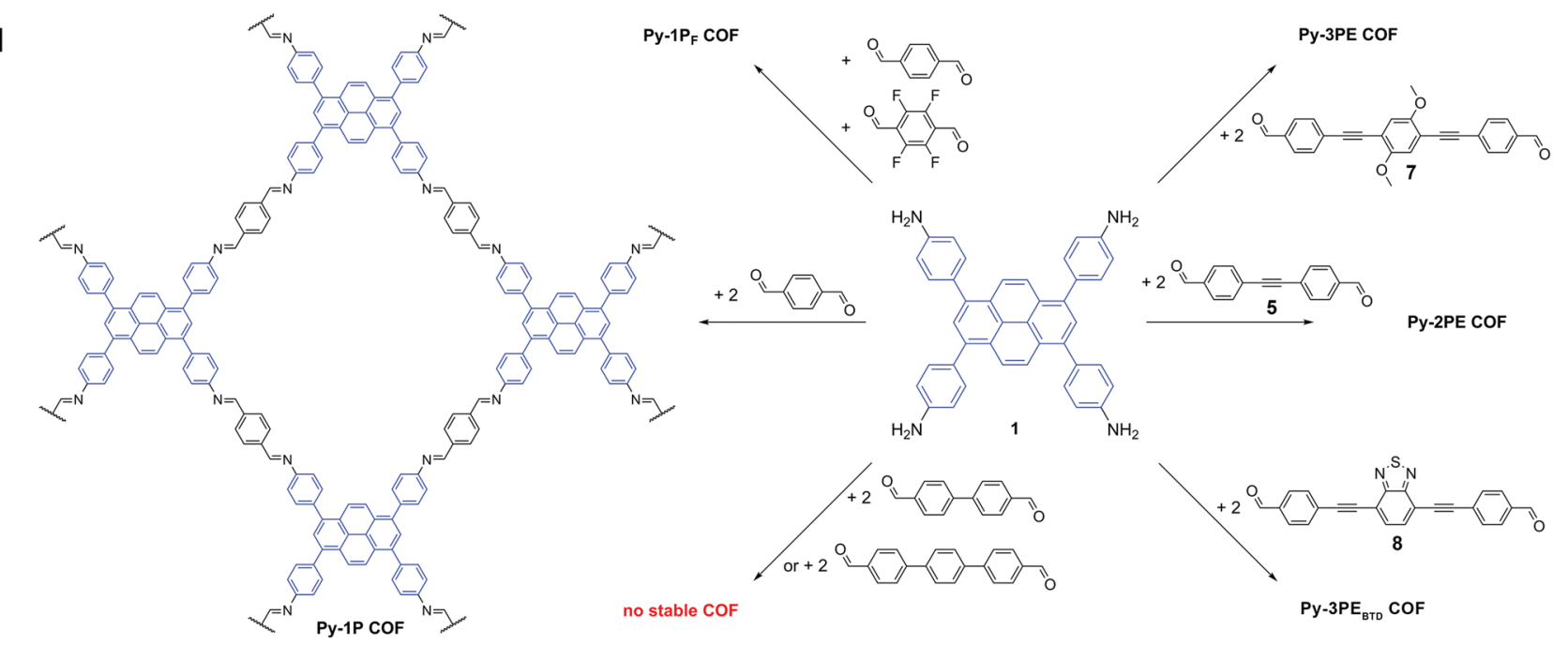

Figure 1. Possible molecular conformations and corresponding stacking arrangements of 1,3,6,8-tetrakis(4-aminophenyl)pyrene when incorporated into a COF ( $\mathrm{C}$, gray; $\mathrm{H}$, white; $\mathrm{N}$, blue). In the "propeller" conformation (a) the normal vectors of the phenylenes describe a circle. These molecules form offset stacks of alternating left- and right-handed propellers, whereby the phenylenes arrange in an edge-on-face sequence. In the "armchair" configuration (b) the normal vectors of the phenylenes point into the same direction. Owing to the reduced steric demands in this configuration the molecules can stack more closely and with reduced lateral offset. (c) Forming defect-free COF domains requires ensuring the same stacking direction of neighboring pyrene stacks. We propose that this can be achieved by synchronizing the orientation of the phenylenes across flat, cofacially stacked bridges. (d) Chemical structures of the building blocks used in the synthesis of the new pyrene-based COFs.

COFs are synthesized via reversible formation of covalent bonds, the most widely applied linkage motifs to date being boronate esters, $^{17-20}$ imines, ${ }^{21-24}$ and hydrazones. ${ }^{25,26}$ In all of these cases, this reversibility of the bond formation under reaction conditions provides the growing COF crystal with a functional self-healing mechanism, a factor of key importance for obtaining a long-range ordered network. ${ }^{27}$ However, as the formation and cleavage of these covalent bonds involve several reaction partners and intermediates, these processes will inherently be more complex and typically slower than for example the coordinative network formation in metal-organic frameworks (MOFs), or the crystallization of small organic molecules, which is driven mainly by Coulomb and dispersion interactions. As a result, crystalline COF structures can be achieved only on very small length scales of typically a few tens to hundred nanometers. If it were possible, however, to direct the attachment of a building block to the growing COF domain such that the correct orientation is highly favored over all other possible attachment geometries, one could expect to obtain enhanced crystallinity within a given number of average attachment and detachment cycles per building block. This could ultimately pave the way for the development of COF single crystals.

We have recently developed a concept for growing highly crystalline 2D COFs that exhibit well-defined hexagonal facets and are devoid of amorphous regions between the individual crystallites. ${ }^{28}$ In this concept, the $3 \mathrm{D}$ molecular conformation generates a uniquely defined docking site for the attachment of a successive COF layer, thus greatly lowering the probability of stacking faults and strained regions due to defects within the individual layers. While we have realized this deterministic approach to COF growth using propellershaped central building blocks, it would be desirable to formulate a geometric concept that imposes less strict boundary conditions on the selection of building blocks. Additionally, enabling significant $\pi$-orbital overlap between extended opto- and electroactive building blocks such as acenes, porphyrins, or tetrathiafulvalenes would be a prerequisite for highly conductive frameworks.

Here, we present a design concept for growing highly crystalline COFs with domain sizes on the order of half a micrometer based on the synchronized offset-stacking of the 


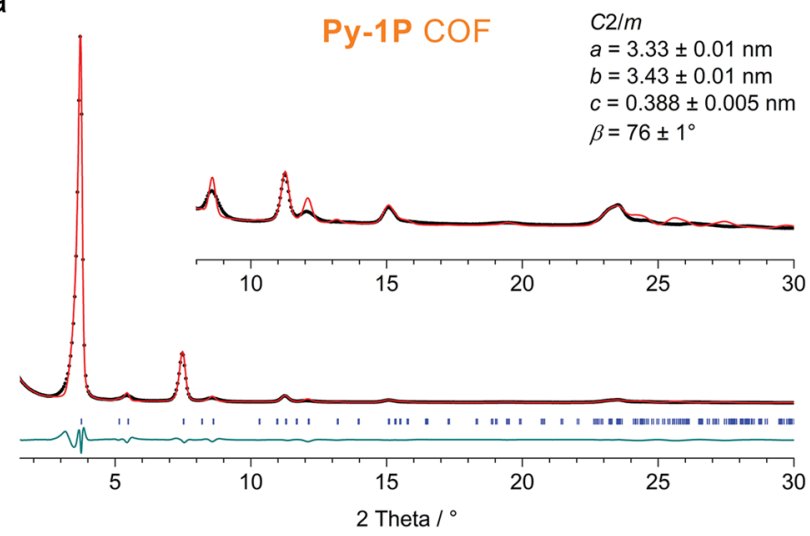

b

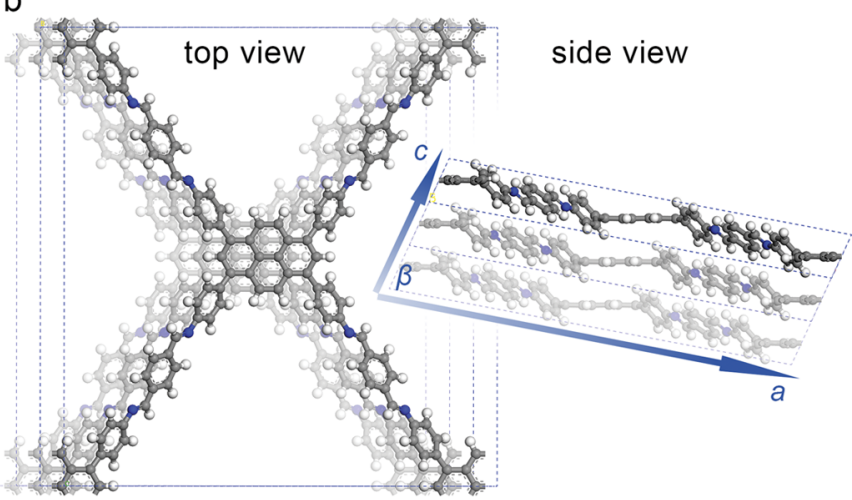

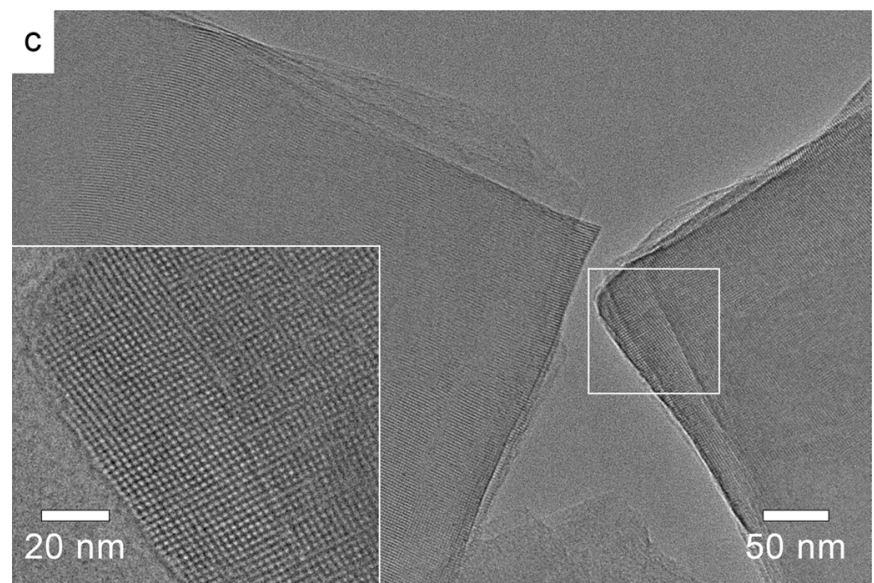

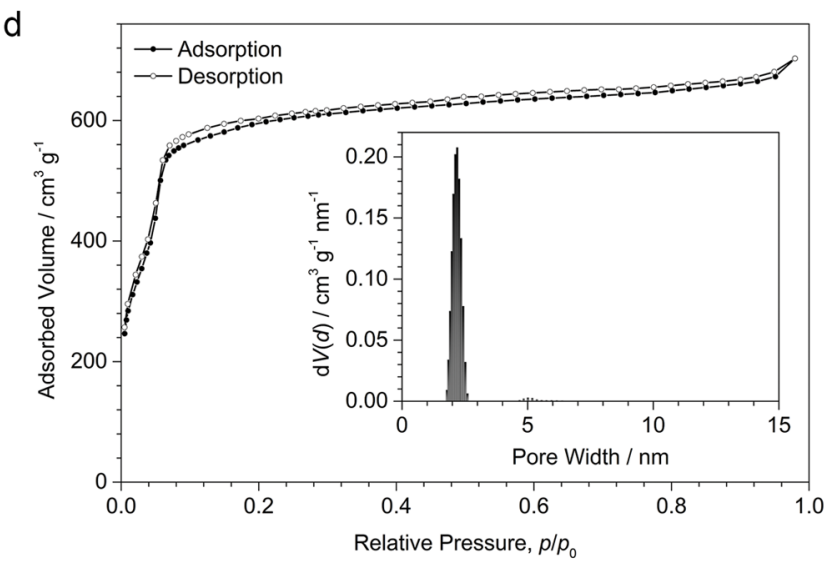

Figure 2. (a) Experimental PXRD pattern (black dots) of the Py-1P COF. Rietveld refinement (red line) provides a very good fit to the experimental data with only minimal differences (the green line shows the difference plot between the experimental PXRD pattern and the one obtained by Rietveld refinement; $\left.R_{w p}=4.91 \%, R_{p}=10.32 \%\right)$. Bragg positions are indicated by blue ticks. Inset, magnified view of the $2 \theta>8^{\circ}$ region. (b) The corresponding unit cell with the viewing direction normal to the pyrene core (left) and onto the side (right). These data are available as Supporting Information. (c) High resolution TEM image showing the large crystal domains of the Py-1P COF. Inset, magnified view showing the pseudoquadratic arrangement of the mesopores. The white frame indicates the magnified area. (d) Nitrogen sorption isotherm recorded at $77 \mathrm{~K}$. Inset, QSDFT calculation using an equilibrium model yields the very narrow pore-size distribution that is anticipated for a fully crystalline lattice.

building blocks. The core moiety of the multidentate building block is hereby allowed to $\pi$-stack, thus providing the framework with a very stable interlayer distance and enabling electronic contact between the layers. The four phenylene substituents on this core serve a dual purpose. They bear the chemical functionality used to cross-link the framework, but also define the magnitude and direction of the offset between two COF layers. In this way, the molecular geometry of the central building block gives rise to a uniquely defined docking site that can guide the attachment of successive layers. Implementing this concept, we synthesized a series of highly crystalline pyrene-based COFs with a slip-stacked quasiquadratic structure. Time-resolved and steady-state optical spectroscopy revealed considerable delocalization of excitations along the $\pi$-stacked columns and, depending on the selection of the building blocks, electronic coupling and charge-transfer transitions across the imine bonds.

\section{RESULTS AND DISCUSSION}

To implement our concept we chose 1,3,6,8-tetrakis(4aminophenyl)pyrene (1) as central building block. Pyrenebased building blocks used in previous COF studies were found to produce appreciably well-ordered networks. ${ }^{2,29,30}$ As is the case for most imine-linked COFs, the amine functionality required to cross-link the network is not directly attached to the core, but added in the form of a 4aminophenyl substituent. In addition to practical reasons such as straightforward synthesis via cross-coupling reactions and a more core-independent reactivity of the aniline groups compared to a directly attached amine, these anilines provide the nonplanarity of the building block that is required to generate a geometric docking site.

Steric repulsion between the phenylene hydrogens and the six hydrogen atoms of the core causes the four phenylenes to be rotated against the pyrene by typically $40-60^{\circ} .^{31}$ In contrast to smaller building blocks such as 1,1,2,2-tetrakis(4aminophenyl)ethene, ${ }^{28}$ the phenylenes on the pyrene are not sterically coupled and thus the molecule can in theory adopt several geometric configurations; the most symmetrical ones are shown in Figures $1 \mathrm{a}$ and $\mathrm{b}$.

In order to study possible stacking modes of the tetraphenylpyrene subunit when incorporated into a COF, we synthesized a molecular model compound $\mathbf{2}$ by imine condensation between 1 and four equivalents of benzaldehyde (see the Supporting Information for experimental details). This model compound was found to crystallize in the 


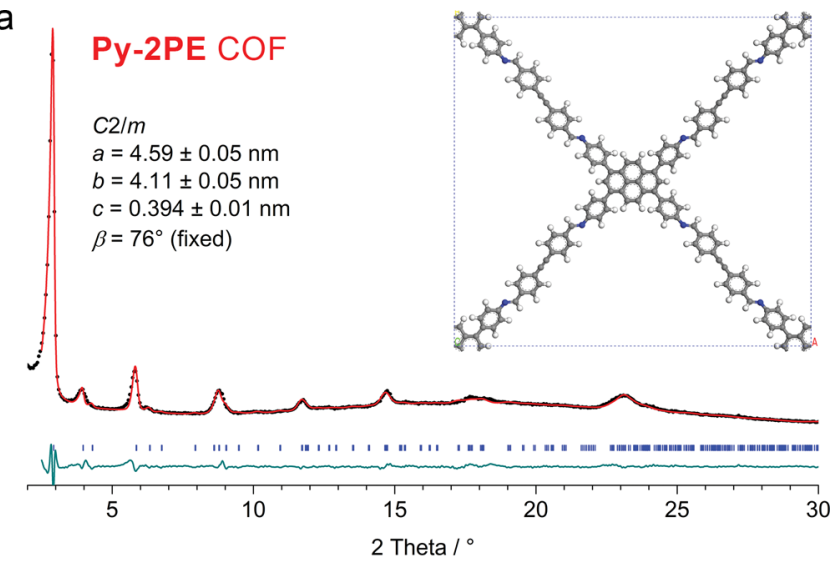

b

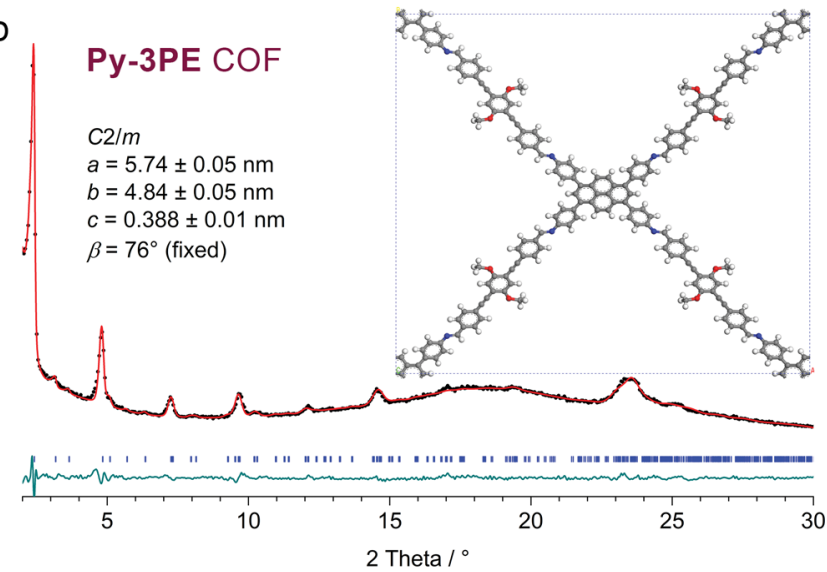

C

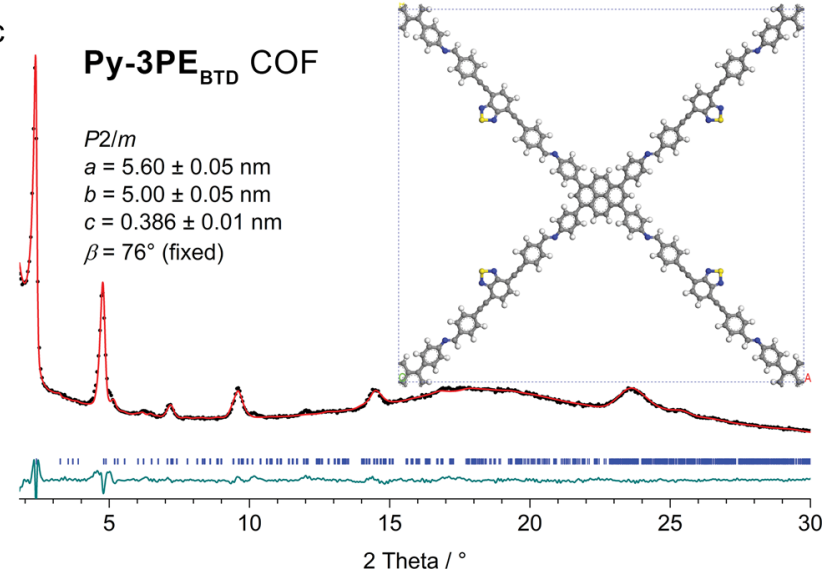

Figure 3. Experimental PXRD patterns (black dots), Pawley-refined patterns (red lines), difference plots (green lines), and Bragg positions (blue ticks) of (a) the Py-2PE, (b) the Py-3PE, and (c) the Py-3PE $\mathbf{B}_{\mathrm{BTD}} \mathrm{COFs}$, respectively. Insets, the corresponding refined unit cells. Compared to the Py-1P COF, the reflection intensities of these COFs appear weaker due to the inclusion of trapped oligomers in the pores that cannot be fully removed without compromising the crystallinity of the framework.

"propeller" configuration also observed for the parent tetraphenylpyrene, ${ }^{31}$ with slipped stacks of alternating rightand left-handed propellers (SI, Figure S2). Because of the sterically demanding edge-on-face configuration of the four phenylenes, the angle $\varphi$ at which the pyrene layers are offset is $48.9^{\circ}$. The average spacing of the pyrenes $d$ in this arrangement is approximately $0.41 \mathrm{~nm}$, considerably larger than typical distances of $\pi$-stacked acenes. ${ }^{11}$

We then simulated a possible COF structure for this stacking motif, linking $\mathbf{1}$ with the smallest aromatic dialdehyde, i.e., terephthalaldehyde (SI, Figure S3a). Balancing the attractive interactions between the pyrene cores and the steric demands of the edge-on-face stacked phenylenes leads to the formation of fairly offset pyrene stacks in the framework (Figure 1a). We find $\varphi=50.9^{\circ}$, and $d=0.42$ $\mathrm{nm}$ for the simulated COF, in good agreement with the experimental parameters for the molecular crystals of 2 .

As this "propeller" structure is not particularly close-packed, we reasoned that there might be another geometric arrangement that could allow for stronger interactions between the COF layers and thus produce a thermodynamically more stable framework. The "armchair" configuration, in which the normal vectors of the four phenylenes point in the same direction, would allow for the cofacial arrangement of both the pyrene cores and the phenylenes (Figure $1 \mathrm{~b}$ ). The distances in this geometry would be determined by the $\pi-\pi$ interactions. Although this molecular configuration has, to the best of our knowledge, not yet been observed in molecular crystals of tetraphenylpyrene derivatives, recent examples of tetra(2-pyridyl)ethene complexes have shown that external interactions, for example the complexation of a transition metal ion, can provide sufficient driving force for the transition from the "propeller" to the "armchair" configuration. $^{32}$

Simulating the above COF with the pyrenes in the "armchair" configuration indeed led to a much closer-packed framework (SI, Figure S3b). Here the pyrenes form slipstacked columns with greatly reduced offset and shorter coreto-core distances of $\varphi=70.3^{\circ}$ and $d=0.39 \mathrm{~nm}$, respectively (Figure 1b).

Comparing the two molecular configurations regarding their ability to direct the attachment of successive COF layers reveals further differences. The "armchair" conformation generates a uniquely defined docking site in which the stacking distance and the lateral offset are defined by the $\pi-\pi$ interactions between the pyrene cores and the phenylenes, respectively (SI, Figure S4b). Here, also the direction of the offset is uniquely defined through the tilt of the phenylenes with respect to the core. In the case of the "propeller" configuration, however, a single building block can only define the stacking distance and the magnitude of the offset. As in this configuration the phenylenes of a single molecule do not possess a preferred direction, the second building block can attach in two positions (SI, Figure S4a). Directional information that can be transferred to successive building blocks via the chains of edge-on-face oriented phenylenes only exists once a dimer has formed.

At this stage, it remains difficult to predict which of the above configurations will be present in our COFs, because this might also be influenced by the choice of the linear building block. As the two conformers stack at very different offset angles, however, the experimentally determined unit cell might allow for conclusions regarding the molecular geometry.

In a defect-free crystal, all pyrene stacks would be offset in the same direction. As the incorporation of a pyrene stack with the "wrong" offset direction would cause immense strain and a large number of defects to compensate it, the synchronization of all pyrene stacks throughout a crystal domain is of key importance. In the "armchair" case, we 
anticipate that this can be achieved if the linear building block is able to transport information about the orientation of the phenylene moieties from one pyrene to its neighbors, ensuring coplanar orientation (Figure 1c). This synchronization mechanism is expected to work well with flat and rigid linear building blocks, such as terephthalaldehyde, whereas twisted molecules such as biphenyl-4,4'-dicarbaldehyde fail to ensure the coplanar orientation of the phenylenes and hence are not expected to form a stable framework. For geometric reasons the "propeller" conformation, on the other hand, cannot incorporate aligned and closely packed linear building blocks. We thus expected it to be less sensitive to the flatness of the linear units, but also less effective for achieving longrange synchronization.

We used the 1,3,6,8-tetrakis(4-aminophenyl)pyrene building block (1) in the solvothermal synthesis of imine-linked COFs in combination with a series of linear dialdehydes (Figure 1d, see the SI for experimental details). Regarding the choice of the building blocks, in view of the above considerations we reasoned that the differences between the twisted biphenyl and terphenyl dialdehydes and their flat ethynylene-bridged counterparts should provide us with clear conclusions about the preferred stacking mode in our COFs. The flat building blocks, and in particular the 2,1,3-benzothiadiazole-substituted 8, would moreover allow us to study the electronic coupling between the pyrene cores and their $\pi$-conjugated electron-rich (7) or -deficient (8) counterparts.

The powder X-ray diffraction pattern of the Py-1P COF exhibits a large number of well-defined reflections and only very weak background, confirming the formation of a highly crystalline framework (Figure $2 \mathrm{a}$ ). In the following we use the term "crystallinity" to refer to the degree of coherent longrange order within a COF domain (as opposed to the domain size). Rietveld refinement using the "armchair" configuration and assuming $\mathrm{C} 2 / \mathrm{m}$ symmetry reproduced the experimental pattern very well and yielded the lattice parameters $a=3.33$ $\pm 0.01 \mathrm{~nm}, b=3.43 \pm 0.01 \mathrm{~nm}, c=0.388 \pm 0.005 \mathrm{~nm}, \beta=$ $76 \pm 1^{\circ}$ (Figure 2b).

Since the unit cell of the Py-1P COF contains a large number of light atoms and the reflections are broadened even for this well-crystallized framework, it was not possible to refine the coordinates of individual atoms and thus directly observe the conformation of the tetraphenylpyrene. Given the differences in stacking behavior between the two possible structures, however, it is possible to draw conclusions on the molecular geometry from the following considerations:

The comparison between the experimental pattern and the theoretical patterns calculated from the two simulated COF structures yields a good match for the "armchair" configuration with only slightly shifted reflection positions, whereas the "propeller" structure does not resemble the experimental pattern very well (Figures S3c and S3d).

The lattice parameters $c$ and $\beta$ (see above), which can be refined with high accuracy from the position and splitting of the double-peak at $2 \theta=23.4^{\circ}$ and which are very sensitive to the packing of the pyrenes, provide a second indication in favor of the less offset and closer-packed "armchair" configuration.

The packing geometry of the "armchair" structure was further confirmed by density functional theory (DFT) calculations using the CASTEP code with the generalizedgradient-approximation (GGA) PBE functional and a correction for dispersion interactions (see the SI for details).
These quantum-mechanical simulations yielded $\beta=75.2^{\circ}$, in very good agreement with the experimental unit cell.

In order to obtain independent experimental evidence for the presence of the "armchair" configuration in the Py-1P COF, we synthesized this framework using a 1:1 mixture of terephthalaldehyde and 2,3,5,6-tetrafluoroterephthalaldehyde. This mixture has been found to add an additional electrostatic stabilization to COFs via the formation face-on-face oriented stacks of alternating fluorinated and nonfluorinated phenylenes. ${ }^{33}$ The PXRD pattern of this Py-1 $\mathbf{P}_{\mathbf{F}}$ COF exhibits the same sequence of reflections and in particular contains the double-peak that stems mainly from the 111 and 001 reflections, here slightly shifted to $23.6^{\circ}$ (SI, Figure S5). The unit cell parameters for this double-layer COF after Rietveld refinement in the space group $P 2 / m$ are $a=3.38 \pm$ $0.02 \mathrm{~nm}, b=3.40 \pm 0.02 \mathrm{~nm}, c=0.772 \pm 0.01 \mathrm{~nm}, \beta=75 \pm$ $2^{\circ}$. The experimental finding that the forced face-to-face arrangement of the bridges in the $\mathbf{P y}-\mathbf{1} \mathbf{P}_{\mathrm{F}}$ COF gives rise to a framework that is almost identical to the nonfluorinated Py1P COF, is another strong indication for the presence of cofacially stacked bridges and hence "armchair"-type pyrenes in our COFs.

In conclusion, based on the above findings we offer strong theoretical and experimental evidence for the presence of the "armchair" configuration of the tetraphenylpyrenes in the Py1P COF. To the best of our knowledge, this is the first time that the crystallization of a COF framework has been observed to change the geometry of one of its constituents into a conformation that does not occur in molecular solids.

Transmission electron microscopy (TEM) images of the Py-1P COF reveal that this material crystallizes in the form of platelets with very large domain sizes of 300-500 nm (Figure $2 c)$. Domains oriented with their crystallographic $a-b$ plane perpendicular to the viewing direction show the highly ordered pseudoquadratic arrangement of the mesopores. Moreover, the individual crystallites feature well-defined facets that are devoid of any visible amorphous regions.

With pore diagonals of 2.00, 2.44, and $2.76 \mathrm{~nm}$ (bridge-tobridge and the two pyrene-to-pyrene distances, respectively) in the refined structure, the Py-1P COF is at the border between micro- and mesoporosity. Its type IV nitrogen sorption isotherm, which is typical for mesoporous materials, exhibits a sharp step and an $\mathrm{H} 1$ hysteresis loop at low $p / p_{0}$ values, confirming the expected small pore size (Figure $2 \mathrm{~d}$ ). The pore diameter calculated by quenched-solid density functional theory (QSDFT) using an equilibrium model and assuming a cylindrical pore geometry is $2.20 \mathrm{~nm}$, in very good agreement with the averaged pore diagonals. The BrunauerEmmett-Teller (BET) surface area of the Py-1P COF is $2210 \pm 50 \mathrm{~m}^{2} \mathrm{~g}^{-1}$ with a total pore volume of $1.09 \pm 0.03$ $\mathrm{cm}^{3} \mathrm{~g}^{-1}$. These values are in excellent agreement with the Connolly surface and total pore volume of $2160 \mathrm{~m}^{2} \mathrm{~g}^{-1}$ and $0.98 \mathrm{~cm}^{3} \mathrm{~g}^{-1}$, respectively, confirming that the pores are open and fully accessible.

The formation of the COF during the solvothermal synthesis proceeds, similar to other imine-linked frameworks, $^{34}$ via an initially formed amorphous network that is converted into the crystalline Py-1P COF over the course of several hours (SI, Figure S7). After $1 \mathrm{~d}$ the main reflections hardly change in intensity any more, indicating nearly full conversion to the crystalline COF. Weaker reflections, however, continue to become more distinct, suggesting an ongoing recrystallization of the material accompanied by 

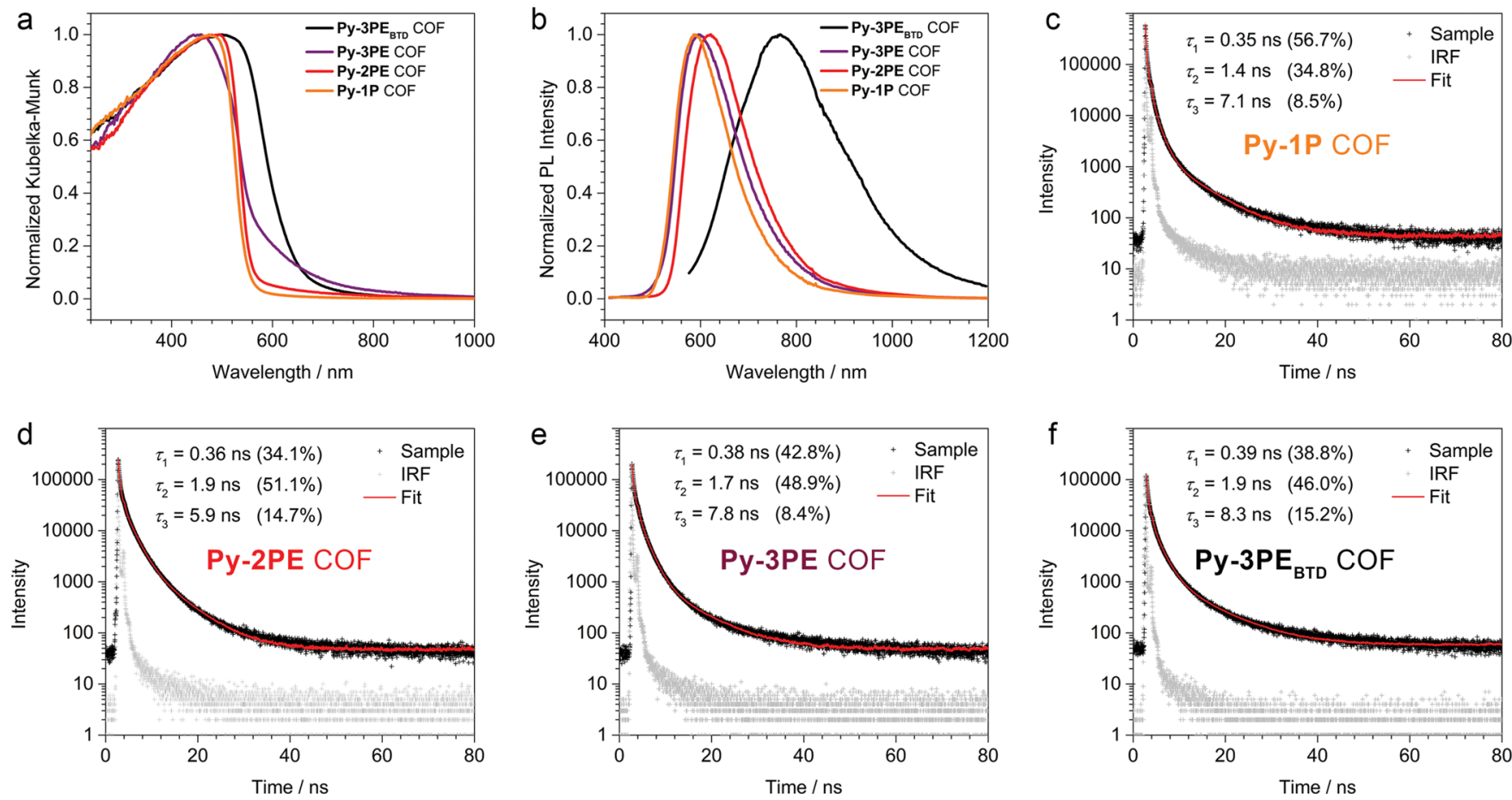

Figure 4. Diffuse reflectance (a) and PL spectra (b) of the four COFs. (c-f) TCSPC traces (black), instrument response functions (IRF, gray), and the corresponding triexponential deconvolution fits of the COFs (red lines). The fraction of collected photons corresponding to the respective lifetimes are given in brackets. Photoexcitation was achieved with a picosecond diode laser at $403 \mathrm{~nm}$.

further improvements in crystal quality. In combination with our lock-and-key design of the building blocks, this mechanism can reliably produce fully crystallized nano- to microcrystalline COFs. Even larger crystals can be anticipated if future improvements in the linkage chemistry could suppress the fast condensation of the amorphous network.

In order to broaden the scope of the above concept we used a series of directly linked and ethynylene-bridged building blocks in the COF syntheses. As expected from the above geometric considerations, the twisted biphenyl (2P) and terphenyl (3P) derived building blocks fail to produce stable crystalline frameworks. The Py-2P COF forms in the reaction solution, but quickly loses its long-range order upon evaporation of the solvent (SI, Figure S6).

If an acetylene bridge, however, is introduced between the phenylenes, the molecule can adopt a planar configuration that should allow for the synthesis of well-ordered "armchair" type frameworks. ${ }^{35}$ Indeed, we were able to obtain crystalline COF materials using the acetylene-bridged building blocks (Figures $3 \mathrm{a}$ and $3 \mathrm{~b}$ ). Pawley refinements carried out in the space group $\mathrm{C} 2 / \mathrm{m}$ with the angle $\beta$ fixed at $76^{\circ}$ (i.e., assuming similar stacking offset as for the Py-1P COF) yielded the lattice parameters $a=4.59 \pm 0.05 \mathrm{~nm}, b=4.11 \pm$ $0.05 \mathrm{~nm}, c=0.394 \pm 0.01 \mathrm{~nm}, \beta=76^{\circ}$ (fixed) and $a=5.74$ $\pm 0.05 \mathrm{~nm}, b=4.84 \pm 0.05 \mathrm{~nm}, c=0.388 \pm 0.01 \mathrm{~nm}, \beta=$ $76^{\circ}$ (fixed) for the Py-2PE and the Py-3PE COFs, respectively.

This concept allows us also to incorporate additional electronic functionality into the building blocks and thus modulate the electronic structure of the resulting frameworks. We demonstrate this by applying a 2,1,3-benzothiadiazolemodified (BTD) version of the 3PE building block. Pawley refinement of the Py-3PE $\mathbf{B}_{\mathbf{B} \text { D }}$ COF in the space group $P 2 / \mathrm{m}$, assuming the most symmetric arrangement of the BTD units in the framework, produced the lattice parameters $a=5.60 \pm$ $0.05 \mathrm{~nm}, b=5.00 \pm 0.05 \mathrm{~nm}, c=0.386 \pm 0.01 \mathrm{~nm}, \beta=76^{\circ}$ (fixed) (Figure 3c).

The new pyrene-based COFs are intensely colored solids ranging from bright orange (Py-1P and Py-2PE COFs) to deep red (Py-3PE $\left.\mathbf{B T D}_{\mathrm{BTD}} \mathrm{COF}\right)$. UV-vis diffuse reflectance spectra corroborate these observations (Figure 4a). Compared to a solution of compound $\mathbf{1}$, the framework absorption edge is red-shifted by about $100-150 \mathrm{~nm}$, indicating considerable electronic delocalization across the pyrene stacks (SI, Figure S12). The Py-1P and Py-2PE COFs feature particularly sharp absorption onsets around $560 \mathrm{~nm}$, whereas the absorption spectrum of the Py-3PE COF is more curved in the normalized representation due to an overlap of the pyrene and the acetylene bridge absorption. In all three cases, the steepest rise in the absorption spectrum is at almost the same wavelength, indicating that the optical transitions are dominated by the central pyrene building block and only slightly affected by the additional $\pi$-conjugation of the acetylenes. This interpretation is supported by the corresponding photoluminescence (PL) spectra (Figure 4b), showing similar emission at around $600 \mathrm{~nm}$.

In order to obtain a better understanding of the electronic processes in these materials, we determined the absolute external photoluminescence quantum yields (PLQYs) using an integrating sphere (SI, Table S2), ${ }^{36,37}$ and studied the PL decay dynamics via time-correlated single photon counting (TCSPC). All three COFs exhibit PLQYs of around $0.2 \%$ and triexponential decay curves with almost identical lifetimes. These results confirm that, irrespective of the length of the $\pi$ conjugated bridge, the electronic processes are dominated by the tetraphenylpyrene moiety.

Placing a strongly electron-withdrawing BTD group into the bridge, however, does change the band gap of the 
material. The red-shifted absorption and emission spectra are indicative of a charge-transfer excitation at energies below the $\pi-\pi^{*}$ transition of the individual building blocks. This is supported by the significantly lower PLQY of the Py-3PE COF. The recombination of the emissive states, however, appears at very similar time constants as for the other three COFs, highlighting the strong influence of the central building block on the optoelectronic properties.

\section{CONCLUSION}

In this study, we used the three-dimensional geometry of a tetraphenylpyrene-derived building block to generate a periodic lattice of synchronized docking sites for the attachment of consecutive COF layers. This way we were able to grow highly crystalline 2D COFs with domain sizes on the order of half a micrometer that feature well-defined facets and are devoid of any observable amorphous regions. In combination with future improvements in the linkage chemistry, this geometric guidance could pave the way for the development of COF single crystals. Our design principle also allows for the simultaneous incorporation of $\pi$-stacked central building blocks and $\pi$-stacked bridges, thus enabling electronic communication between all subunits of the framework. Studying the optoelectronic properties of a series of pyrene-based COFs revealed significant delocalization of excitations along the pyrene stacks and showed that chargetransfer excitations are possible across the imine bonds. The frameworks developed in this study can serve as a blueprint for designing a broad range of tailor-made 2D COFs with extended $\pi$-conjugated building blocks for applications in photocatalysis and optoelectronics.

\section{ASSOCIATED CONTENT}

\section{S Supporting Information}

The Supporting Information is available free of charge on the ACS Publications website at DOI: 10.1021/jacs.6b09787.

Experimental methods, synthetic procedures, and additional structural and spectroscopic data (PDF) Crystal data (CIF)

\section{AUTHOR INFORMATION}

\section{Corresponding Author}

*bein@lmu.de

\section{ORCID ${ }^{\circ}$}

Florian Auras: 0000-0003-1709-4384

Paul Knochel: 0000-0001-8167-272X

Timothy Clark: 0000-0001-7931-4659

\section{Notes}

The authors declare no competing financial interest.

\section{ACKNOWLEDGMENTS}

The authors are grateful for funding from the German Science Foundation (DFG; Research Cluster NIM) and the Free State of Bavaria (Research Network SolTech). The research leading to these results has received funding from the European Research Council under the European Union's Seventh Framework Programme (FP7/2007-2013)/ERC Grant Agreement No. 321339.

\section{REFERENCES}

(1) Doonan, C. J.; Tranchemontagne, D. J.; Glover, T. G.; Hunt, J. R.; Yaghi, O. M. Nat. Chem. 2010, 2, 235.

(2) Rabbani, M. G.; Sekizkardes, A. K.; Kahveci, Z.; Reich, T. E.; Ding, R.; El-Kaderi, H. M. Chem. - Eur. J. 2013, 19, 3324.

(3) Ding, S.-Y.; Gao, J.; Wang, Q.; Zhang, Y.; Song, W.-G.; Su, C.Y.; Wang, W. J. Am. Chem. Soc. 2011, 133, 19816.

(4) Lin, S.; Diercks, C. S.; Zhang, Y.-B.; Kornienko, N.; Nichols, E. M.; Zhao, Y.; Paris, A. R.; Kim, D.; Yang, P.; Yaghi, O. M.; Chang, C. J. Science 2015, 349, 1208.

(5) Dogru, M.; Handloser, M.; Auras, F.; Kunz, T.; Medina, D.; Hartschuh, A.; Knochel, P.; Bein, T. Angew. Chem., Int. Ed. 2013, 52, 2920.

(6) Calik, M.; Auras, F.; Salonen, L. M.; Bader, K.; Grill, I.; Handloser, M.; Medina, D. D.; Dogru, M.; Löbermann, F.; Trauner, D.; Hartschuh, A.; Bein, T. J. Am. Chem. Soc. 2014, 136, 17802.

(7) Cai, S.-L.; Zhang, Y.-B.; Pun, A. B.; He, B.; Yang, J.; Toma, F. M.; Sharp, I. D.; Yaghi, O. M.; Fan, J.; Zheng, S.-R.; Zhang, W.-G.; Liu, Y. Chem. Sci. 2014, 5, 4693.

(8) Côte, A. P.; Benin, A. I.; Ockwig, N. W.; O’Keeffe, M.; Matzger, A. J.; Yaghi, O. M. Science 2005, 310, 1166.

(9) Spitler, E. L.; Colson, J. W.; Uribe-Romo, F. J.; Woll, A. R.; Giovino, M. R.; Saldivar, A.; Dichtel, W. R. Angew. Chem., Int. Ed. 2012, 51, 2623.

(10) Jin, S.; Ding, X.; Feng, X.; Supur, M.; Furukawa, K.; Takahashi, S.; Addicoat, M.; El-Khouly, M. E.; Nakamura, T.; Irle, S.; Fukuzumi, S.; Nagai, A.; Jiang, D. Angew. Chem., Int. Ed. 2013, 52, 2017.

(11) Patwardhan, S.; Kocherzhenko, A. A.; Grozema, F. C.; Siebbeles, L. D. A. J. Phys. Chem. C 2011, 115, 11768.

(12) Medina, D. D.; Werner, V.; Auras, F.; Tautz, R.; Dogru, M.; Schuster, J.; Linke, S.; Döblinger, M.; Feldmann, J.; Knochel, P.; Bein, T. ACS Nano 2014, 8, 4042.

(13) Guo, J.; Xu, Y.; Jin, S.; Chen, L.; Kaji, T.; Honsho, Y.; Addicoat, M. A.; Kim, J.; Saeki, A.; Ihee, H.; Seki, S.; Irle, S.; Hiramoto, M.; Jiang, D. Nat. Commun. 2013, 4, 2736.

(14) Bertrand, G. H. V.; Michaelis, V. K.; Ong, T.-C.; Griffin, R. G.; Dinca, M. Proc. Natl. Acad. Sci. U. S. A. 2013, 110, 4923.

(15) Ding, H.; Li, Y.; Hu, H.; Sun, Y.; Wang, J.; Wang, C.; Wang, C.; Zhang, G.; Wang, B.; Xu, W.; Zhang, D. Chem. - Eur. J. 2014, 20, 14614.

(16) Jin, S.; Sakurai, T.; Kowalczyk, T.; Dalapati, S.; Xu, F.; Wei, H.; Chen, X.; Gao, J.; Seki, S.; Irle, S.; Jiang, D. Chem. - Eur. J. 2014, 20, 14608.

(17) Spitler, E. L.; Dichtel, W. R. Nat. Chem. 2010, 2, 672.

(18) Spitler, E. L.; Koo, B. T.; Novotney, J. L.; Colson, J. W.; Uribe-Romo, F. J.; Gutierrez, G. D.; Clancy, P.; Dichtel, W. R. J. Am. Chem. Soc. 2011, 133, 19416.

(19) Feng, X.; Honsho, Y.; Saengsawang, O.; Liu, L.; Wang, L.; Saeki, A.; Irle, S.; Seki, S.; Dong, Y.; Jiang, D. Adv. Mater. 2012, 24, 3026.

(20) Smith, B. J.; Dichtel, W. R. J. Am. Chem. Soc. 2014, 136, 8783.

(21) Uribe-Romo, F. J.; Hunt, J. R.; Furukawa, H.; Klöck, C.; O'Keeffe, M.; Yaghi, O. M. J. Am. Chem. Soc. 2009, 131, 4570.

(22) Wan, S.; Gándara, F.; Asano, A.; Furukawa, H.; Saeki, A.; Dey, S. K.; Liao, L.; Ambrogio, M. W.; Botros, Y. Y.; Duan, X.; Seki, S.; Stoddart, J. F.; Yaghi, O. M. Chem. Mater. 2011, 23, 4094.

(23) Kandambeth, S.; Shinde, D. B.; Panda, M. K.; Lukose, B.; Heine, T.; Banerjee, R. Angew. Chem., Int. Ed. 2013, 52, 13052.

(24) Dalapati, S.; Addicoat, M.; Jin, S.; Sakurai, T.; Gao, J.; Xu, H.; Irle, S.; Seki, S.; Jiang, D. Nat. Commun. 2015, 6, 7786.

(25) Uribe-Romo, F. J.; Doonan, C. J.; Furukawa, H.; Oisaki, K.; Yaghi, O. M. J. Am. Chem. Soc. 2011, 133, 11478.

(26) Stegbauer, L.; Schwinghammer, K.; Lotsch, B. V. Chem. Sci. 2014, 5, 2789.

(27) Calik, M.; Sick, T.; Dogru, M.; Döblinger, M.; Datz, S.; Budde, H.; Hartschuh, A.; Auras, F.; Bein, T. J. Am. Chem. Soc. 2016, 138, 1234. 
(28) Ascherl, L.; Sick, T.; Margraf, J. T.; Lapidus, S. H.; Calik, M.; Hettstedt, C.; Karaghiosoff, K.; Döblinger, M.; Clark, T.; Chapman, K. W.; Auras, F.; Bein, T. Nat. Chem. 2016, 8, 310.

(29) Chen, X.; Huang, N.; Gao, J.; Xu, H.; Xu, F.; Jiang, D. Chem. Commun. 2014, 50, 6161.

(30) Wu, Y.; Xu, H.; Chen, X.; Gao, J.; Jiang, D. Chem. Commun. 2015, 51, 10096.

(31) Feng, X.; Tomiyasu, H.; Hu, J.-Y.; Wei, X.; Redshaw, C.; Elsegood, M. R. J.; Horsburgh, L.; Teat, S. J.; Yamato, T. J. Org. Chem. 2015, 80, 10973.

(32) D’Alessandro, D. M.; Keene, F. R.; Steel, P. J.; Sumby, C. J. Aust. J. Chem. 2003, 56, 657.

(33) Chen, X.; Addicoat, M.; Irle, S.; Nagai, A.; Jiang, D. J. Am. Chem. Soc. 2013, 135, 546.

(34) Smith, B. J.; Overholts, A. C.; Hwang, N.; Dichtel, W. R. Chem. Commun. 2016, 52, 3690.

(35) Nafe, J.; Herbert, S.; Auras, F.; Karaghiosoff, K.; Bein, T.; Knochel, P. Chem. - Eur. J. 2015, 21, 1102.

(36) de Mello, J. C.; Wittmann, H. F.; Friend, R. H. Adv. Mater. 1997, 9, 230.

(37) Fernandez, S.; Ganiek, M. A.; Karpacheva, M.; Hanusch, F. C.; Reuter, S.; Bein, T.; Auras, F.; Knochel, P. Org. Lett. 2016, 18, 3158. 